\title{
Effect of Suture on Mechanical Properties of Stitched Thermal Protection Sandwich
}

\author{
Cong Lin ${ }^{a}$, Fanchun Lee ${ }^{a}{ }^{\circledR} *$, Taohua Xue \\ ${ }^{a}$ Ship Architecture and Ocean Engineering College, Dalian Maritime University, Da Lian116026, China
}

Received: July 20, 2019; Revised: September 20, 2019; Accepted: October 28, 2019

\begin{abstract}
This paper investigates the tensile properties and bending performances of stitched thermal protection structure in engineering, and the effect of stitching density on mechanical properties, via a model of ceramic-aerogel stitched sandwich. With the help of ANSYS, titanium alloy bottom plate was added to the bottom surface of the thermal protection structure, which was regarded as the protected body, and the two were synergistically deformed. The validity of the entire model was verified. The distribution of stress field inside the structure was determined by substructure precise analysis method and the law of the finite element solution was given. The results show that interlaminar performance of the stitched sandwich is improved by $30 \%$ on average under tensile or four-point bending conditions.
\end{abstract}

Keywords: thermal protection structure; stitched sandwich; tensile properties; bending performance; finite element analysis.

\section{Introduction}

The space flight orbiter is subjected to aerodynamic action during the launch/re-entry phase.To protect the fuselage and the internal structure temperature within an acceptable range, a thermal protection structure is required on the surface of the body. The thermal protection structure should also consider the impact of its own weight on the aircraft while ensuring flight safety. The ceramic-aerogel thermal protection structure of the research object in this paper is a composite sandwich comprised of two thin ceramic panels and an aerogel core. The composite sandwich panel is a typical multi-stage structural integrated thermal protection structure, which is laminated ${ }^{1}$. The ceramic thin panel has a certain tensile strength, and the aerogel has the characteristics of low density and small heat transfer coefficient.The sandwich core is heat insulating material, so that the overall structure has low thermal conductivity and high heat capacity, and has good heat insulation performance. However, the connection between the structural layers and the guarantee of the connection strength are the main problems $\mathrm{s}^{2-3}$. Stitched sandwich is developed based on traditional sandwich; suture along the thickness direction of the structure can effectively overcome the defect of easy delamination of traditional laminate structure. Stitching improvesinterlayer performance and impact resistance of laminate structure ${ }^{4}$.This thermal protection sandwich structure is mainly used to solve the thermal protection problem of high-speed aircraft.
To study the mechanical properties of the stitched structure and the laminate, a lot of work has been done by scholars at home and abroad.The suturewas originally applied to laminates. Liu, Fracesconi and Lopresto ${ }^{5-7}$ conducted experiments and simulation studies on suture laminates. The results showed that suture could effectively reduce the lamination area and the superiority of suture lines was obvious in highthickness structures; Mouritz ${ }^{8-9}$ summarized the literature on the mechanical properties of a large number of stitched reinforced laminates, and found that the stiffness makes the structural stiffness strength and fatigue resistance range from $10 \%$ to $20 \%$. Xie et al ${ }^{10-11}$ studied the influence of the main structural parameters of thermal protection structures on structural properties, and provided clear ideas for the finite element analysis of thermal protection structures. Han and $\mathrm{Xitao}^{12-13}$ used finite element software for simulation research and compared with the experimental results; the experimental results agree well with the simulation results, the sutures improve the defects of the panel delamination and the suture has a significant influence on the in-plane Young's modulus.

The way to improve the performance of the sandwich structure is not only the suture stitching: Shi,Chen and others ${ }^{14}$ have compared the load-displacement curves and failure modes of the toughened and un-toughened specimens through the three-point bending test. The mechanical properties such as flexural strength and energy absorption of the core board are increased by at least $14.06 \%$ and $61.53 \%$ respectively compared with the un-toughened sandwich panels; Vaidya et al. used metal needles to reinforce the foam core composite skin sandwich panel for impact. 
Further, bending tests show that it has good impact resistance and delamination resistance after reinforcement between layers ${ }^{15-17}$.Virakthi, Selverand Raeisi ${ }^{18-20}$ used Z-pin reinforcement structures and performed modelling analysis and impact and bending tests. The results showed that the interfacial adhesion properties were improved.

From the above paragraphs, it can be seen that stitching can effectively resist delamination failure of high-thickness sandwich structures, especially fracture and degumming. Researchers ${ }^{15,16,18,19,20}$ pay attention to interlaminar delamination, but there is a spot of research on changes in in-plane mechanical properties after suture. Based on finite element software platform, this paper explores the tensile properties and bending performance of stitched thermal protection structure through theoretical derivation, experimental model verification and multi-order simulation analysis.

\section{Basic equations for the sandwich}

The thermal protection structure is a sandwich consisting of two layers of panels with smaller thickness and one layer of light sandwich with larger thickness, and the planar rigidity of the panels are larger.The middle surface of the structure is $X Y$ plane, the $Z$ axis is downward, the thickness of the upper and lower panels is $t$, and the thickness of the clamping core ish.According to shear theory ${ }^{21}$, several assumptions are put forward for sandwich panels:

1.The thickness of the panel is small compared with the thickness of the entire interlayer, soit is treated as a film to withstand the in-plane force $\sigma_{x i}, \sigma_{y i^{\prime}} \tau_{x y i}$ (the stress components in different layers: $i=1,2 . i=1$ refers to the upper panel, $i=2$ refers to the lower panel) and is evenly distributed along the thickness.

2.The core is soft, ignoring the stress components parallel to the XY plane in the core, i.e. $\sigma_{x c}=\sigma_{y c}=\tau_{x y c}=0$ (the stress components in the core).

3 .When the sandwich is bent, the upper and lower panels are bent in the same direction, assuming that the $\sigma_{z}$ (the stress component along Z-direction)of the entire structure is 0 .

Take the element in the core, the equilibrium equation $\operatorname{are}^{21}$ :

$$
\left\{\begin{array}{l}
\frac{\partial \sigma_{x}}{\partial x}+\frac{\partial \tau_{x y}}{\partial y}+\frac{\partial \tau_{x z}}{\partial z}=0 \\
\frac{\partial \tau_{x y}}{\partial x}+\frac{\partial \sigma_{y}}{\partial y}+\frac{\partial \tau_{y z}}{\partial z}=0 \\
\frac{\partial \tau_{x z}}{\partial x}+\frac{\partial \tau_{y z}}{\partial y}+\frac{\partial \sigma_{z}}{\partial z}=0
\end{array}\right.
$$

where $\sigma_{\mathrm{x}}, \sigma_{\mathrm{y}}, \sigma_{\mathrm{z}}, \tau_{\mathrm{xy}}, \tau_{\mathrm{yz}}, \tau_{\mathrm{xz}}$ are the stress components in the core layer.

According to Hypothesis 2, the equation can be simplified to

$$
\frac{\partial \tau_{x z}}{\partial z}=0 \quad \frac{\partial \tau_{y z}}{\partial z}=0
$$

When the structure is subjected to the lateral load $q$, lateral shear forces $N_{x z}$ and $N_{y z}$ are generated, thereby:

$$
\tau_{x z}=\frac{N_{x z}}{h+t} \quad \tau_{y z}=\frac{N_{y z}}{h+t}
$$

If the shear modulus of the core is $G_{c}$, the shear strain are:

$$
\left\{\begin{array}{l}
\gamma_{x z}=\frac{\tau_{x z}}{G_{c}}=\frac{N_{x z}}{G_{c}(h+t)} \\
\gamma_{y z}=\frac{\tau_{y z}}{G_{c}}=\frac{N_{y z}}{G_{c}(h+t)}
\end{array}\right.
$$

Geometric equations:

$$
\gamma_{x z}=\frac{\partial \omega}{\sigma x}+\frac{\sigma u}{\sigma z} \quad \gamma_{\gamma z}=\frac{\sigma \omega}{\sigma y}+\frac{\sigma v}{\sigma z}
$$

where $u$ is the generalized displacement in theX-direction of the panel, $v$ is the generalized displacement in the Y-direction of the panel, and $\omega$ is the deflection.

Substituting (4) into (5) and deforming:

$$
\left\{\begin{array}{l}
\frac{\partial u}{\partial z}=-\left[\frac{\partial \omega}{\partial x}-\frac{N_{x z}}{G_{c}(h+t)}\right] \\
\frac{\partial v}{\partial z}=-\left[\frac{\partial \omega}{\partial y}-\frac{N_{y z}}{G_{c}(h+t)}\right]
\end{array}\right.
$$

Integrate $z$ :

$$
\left\{\begin{array}{l}
u=-z\left[\frac{\partial \omega}{\partial x}-\frac{N_{x z}}{G_{c}(h+t)}\right] \\
v=-z\left[\frac{\partial \omega}{\partial y}-\frac{N_{y z}}{G_{c}(h+1)}\right]
\end{array}\right.
$$

Introduce equations $\varphi_{x}=\frac{\partial \omega}{\partial x}-\frac{N_{x z}}{G_{c}(h+t)}, \varphi_{y}=\frac{\partial \omega}{\partial y}-\frac{N_{y z}}{G_{c}(h+t)}$

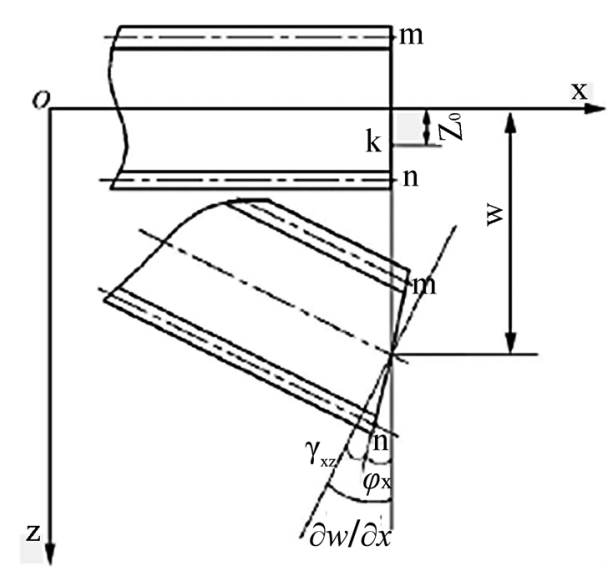

Figure 1. Deformation of bending section of sandwich structure. 
Then the bending section deformation of the shearing theory sandwich panel is shown in Figure1.

Under the transverse load $q$, the transverse balance equations of the sandwich panel are the same as the thin plate balance equations. $M_{x}, M_{y}$ and $M_{x y}$ are the bending moments.

$$
\left\{\begin{array}{l}
\frac{\partial M_{x}}{\partial x}+\frac{\partial M_{x y}}{\partial y}-N_{x z}=0 \\
\frac{\partial M_{y}}{\partial y}+\frac{\partial M_{x y}}{\partial x}-N_{y z}=0 \\
\frac{\partial N_{x z}}{\partial x}+\frac{\partial N_{y z}}{\partial y}+q=0
\end{array}\right.
$$

Since the panel of the sandwich only bears the bending moment, it is assumed ${ }^{21}$ that the stressesare evenly distributed along the thickness, and the bending moment of the sandwich panel can be seen in Figure 2.

$$
\begin{aligned}
M_{x}= & \frac{1}{2}(h+t) t \sigma_{x 1}-\frac{1}{2}(h+t) t \sigma_{x 2} \\
& =\frac{1}{2}(h+t) t\left(\sigma_{x 1}-\sigma_{x 2}\right)
\end{aligned}
$$

We canobtain equations from from above same reason:

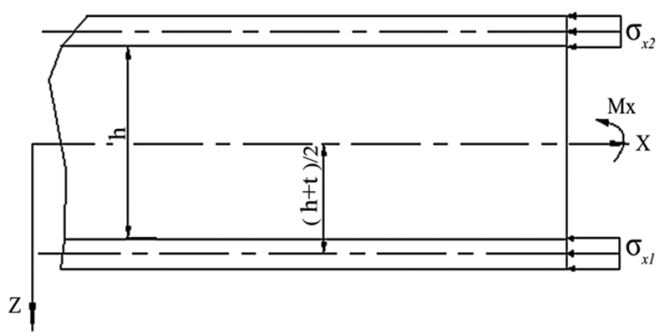

Figure 2. Bending moment of sandwich structure.

$$
\begin{gathered}
M_{y}=\frac{1}{2}(h+t) t\left(\sigma_{y 1}-\sigma_{y 2}\right) \\
M_{x y}=\frac{1}{2}(h+t) t\left(\sigma_{x y 1}-\sigma_{x y 2}\right)
\end{gathered}
$$

The ceramic panel is an isotropic material with the following stresses

$$
\left\{\begin{array}{l}
\sigma_{x i}=\frac{E f}{1-v_{f}^{2}}\left(\varepsilon_{x i}+v_{f} \varepsilon_{y i}\right)=\frac{E_{f}}{1-v_{f}^{2}}\left(\frac{\partial u_{i}}{\partial x}+v_{f} \frac{\partial v i}{\partial y}\right) \\
\sigma_{y i}=\frac{E_{f}}{1-v_{f}^{2}}\left(\varepsilon_{y i}+v_{f} \varepsilon_{x i}\right)=\frac{E_{f}}{1-v_{f}^{2}}\left(\frac{\partial u_{i}}{\partial x}+v_{f} \frac{\partial v_{i}}{\partial y}\right) \\
\tau_{x y i}=\frac{E_{f}}{2\left(1+v_{f}\right)} \gamma_{x y i}=\frac{E_{f}}{2\left(1+v_{f}\right)}\left(\frac{\partial u_{i}}{\partial y}+\frac{\partial v_{i}}{\partial x}\right)(i=1,2)
\end{array}\right.
$$

where $E_{f}$ is Young's modulus, $v_{f}$ is Poisson's ratio.
The displacement at the middle surface of the upper and lower panels are:

$$
\begin{aligned}
& u_{1}=-\frac{1}{2}(h+t) \varphi_{x} \quad u_{2}=\frac{1}{2}(h+t) \varphi_{x} \\
& v_{1}=-\frac{1}{2}(h+t) \varphi_{y} \quad v_{2}=\frac{1}{2}(h+t) \varphi_{y}
\end{aligned}
$$

Substituting the formulas (12-14) into the formulas (9-11):

$$
\left\{\begin{array}{l}
M_{x}=-D\left(\frac{\partial \varphi_{x}}{\partial x}+v_{f} \frac{\partial \varphi_{y}}{\partial y}\right) \\
M_{y}=-D\left(\frac{\partial \varphi_{y}}{\partial y}+v_{f} \frac{\partial \varphi_{x}}{\partial x}\right) \\
M_{x y}=-\frac{1}{2} D\left(1-v_{f}\right)\left(\frac{\partial \varphi_{x}}{\partial y}+\frac{\partial \varphi_{y}}{\partial x}\right)
\end{array}\right.
$$

where $\mathrm{D}$ is the bending stiffness of the sandwich panel, $D=\frac{E_{f}(h+t)^{2} t}{2\left(1-v^{2}\right)}$ 。

$$
N_{x z}=C\left(\frac{\partial \omega}{\partial x}-\varphi_{x}\right) \quad N_{y z}=C\left(\frac{\partial \omega}{\partial y}-\varphi_{y}\right)
$$

$\mathrm{C}$ is the core shear stiffness, $C=G_{c}(h+t)$ 。

Substituting equations (15-16) into equations (8):

$$
\left\{\begin{array}{l}
D\left(\frac{\partial^{2} \varphi_{x}}{\partial x^{2}}+\frac{1-v_{f}}{2} \frac{\partial^{2} \varphi x}{\partial y^{2}}+\frac{1+v_{f}}{2} \frac{\partial^{2} \varphi_{y}}{\partial x \partial y}\right)+C\left(\frac{\partial \omega}{\partial x}-\varphi_{x}\right)=0 \\
D\left(\frac{\partial^{2} \varphi}{\partial y^{2}}+\frac{1-v_{f}}{2} \frac{\partial^{2} \varphi y}{\partial x^{2}}+\frac{1+v_{f}}{2} \frac{\partial^{2} \varphi_{y}}{\partial x \partial y}\right)+C\left(\frac{\partial \omega}{\partial y}-\varphi y\right)=0 \\
C\left(\frac{\partial^{2} \omega}{\partial x^{2}}+\frac{\partial^{2} \omega}{\partial y^{2}}-\frac{\partial \varphi x}{\partial x}-\frac{\partial \varphi_{y}}{\partial y}\right)+q=0
\end{array}\right.
$$

Formulas (17) can be used to solve the three generalized displacements: $\varphi_{x}, \varphi_{y}$ and $\omega$ that can be computed, and then the quantities of $M_{x}, M_{x y}, M_{y}, N_{\mathrm{xz}}$ and $N_{\mathrm{yz}}$ can be obtained and finally, the stress and strain distribution of the sandwich can be obtained.

\section{Test Piece Results}

Four-point bending test was carried out using an Instron 10t mechanical property- testing machine. The mechanical properties of stitched thermal protection structure specimens were compared with the finite element model to verify the validity of the model. The length $\mathrm{L}$ of the thermal protection structure specimen is $300 \mathrm{~mm}$, the width $\mathrm{W}$ is $60 \mathrm{~mm}$, and the total thickness $\mathrm{D}$ is 12 $\mathrm{mm}$. The titanium alloy plate is $600 \mathrm{~mm} \times 70 \mathrm{~mm} \times 5.5 \mathrm{~mm}$. The panel has the same length and width as the core layer, wherein the upper and lower panels have a thickness of $1 \mathrm{~mm}$ and are symmetrical about the core material, and the core layer has a thickness of $10 \mathrm{~mm}$. 
The thermal protection structure was bonded to the titanium alloy base plate by silicone rubber. The experimental design load was $10 \mathrm{KN}$, with $1 \mathrm{KN}$ as the first grade, and the loading rate was $1 \mathrm{~mm} / \mathrm{min}$. The load was kept for 1 min after the loading of each grade was completed. The whole test was completedas per ASTM D6272-17 standard requirements. The position of strain gauge is shown in Figure 3, and the test design drawing and testloading device are shown in Figure 4.

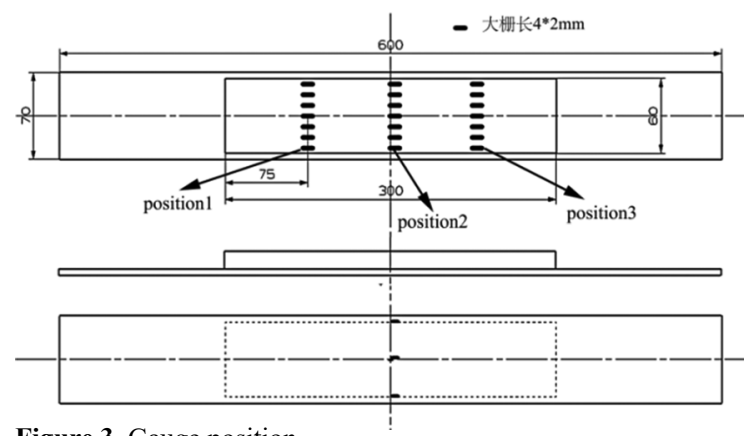

Figure 3. Gauge position.

Considering the influence of temperature, the test was carried out at room temperature. At the 9 th grade $(9 \mathrm{KN})$, the upper panel was wrinkled and cracked. By the time the ninth load is maintained, the compressive strain at the centre of the upper panel reaches $-3 \times 10^{-3}$ and the values of the three strain gauges are close to $-4 \times 10^{-3}$. At this time, the maximum strain of titanium alloy bottom plate is about $4 \times 10^{-3}$, the displacement of pressure head is about $18 \mathrm{~mm}$, and the maximum deflection at the centre of titanium alloy is about $50 \mathrm{~mm}$.

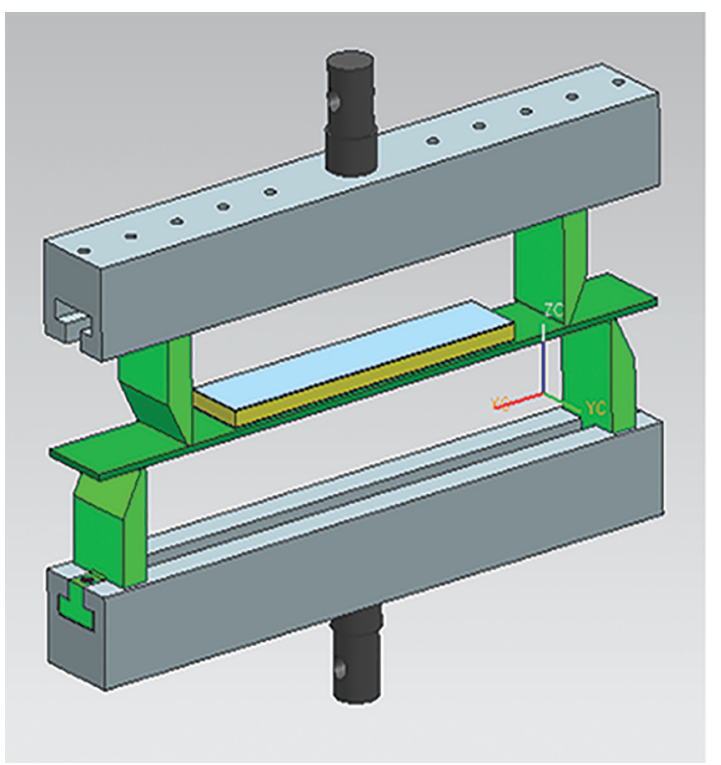

Figure 4. Test schematic diagram and test loading device.
The changing trend of strain with loading step at three points of the upper panel of the suture reinforced thermal protection structure under bending load is shown in Figure 5. Selecting a fixed time point can find that the strain increases from position 1 to position 2 and decreased from position 2 to position3.

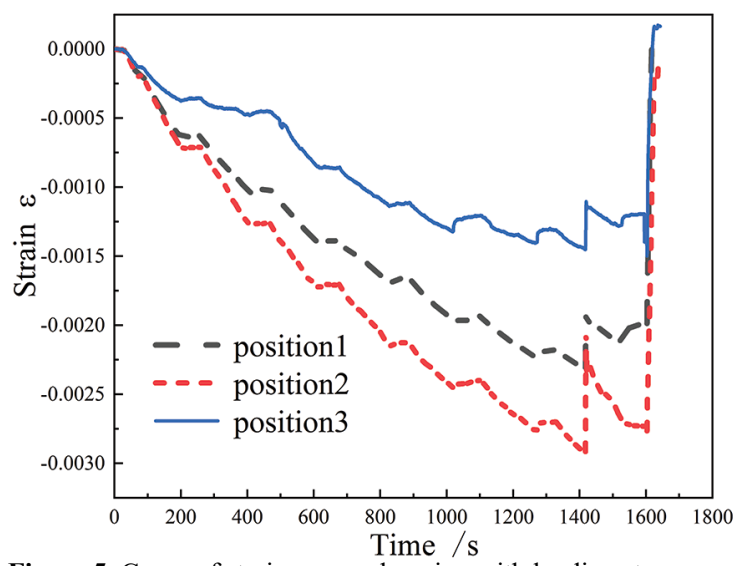

Figure 5. Curve of strain gauge changing with loading step.

\section{Finite Element Model and Model Verification}

The traditional angle suture can effectively increase the number of sutures through the cross arrangement of sutures, but the cross suture at the same position will make the sutures affect each other when transmitting force, and the overall performance is difficult to ensure. Considering the convenience of modelling, the intuition of the model and the convergence of the results, the thermal protection structure is integrally stitched, and the stitching is set to be perpendicular to the plane.

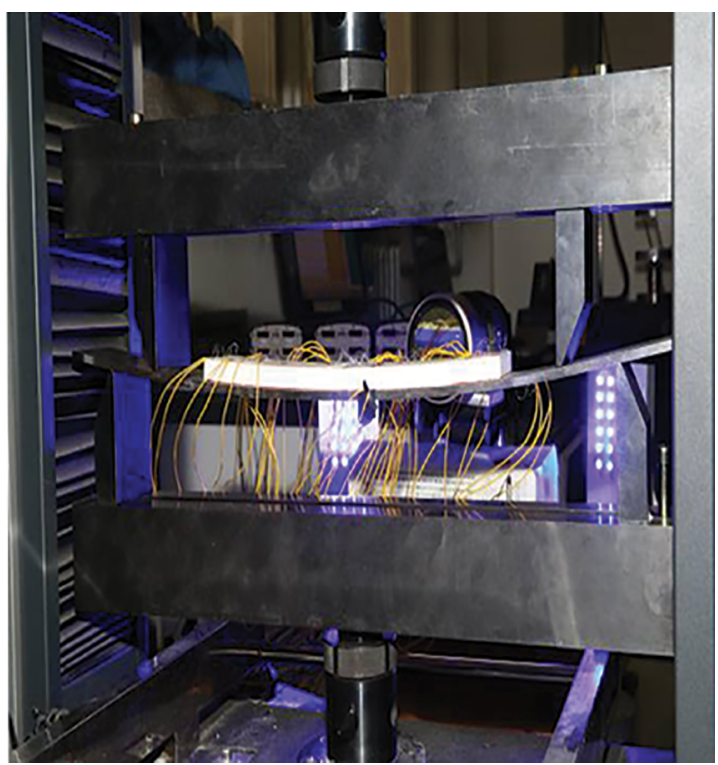


Although suture parameters include many factors such as material parameters, suture diameter and density, suture density is the most influential factor in practical engineering problems ${ }^{22}$.To simplify the simulation steps, expressing suture density with needle pitch, row spacing and suture angle was equivalent to considering all suture parameters.

The specificproperties of the materials are measured through experiments, and the basic properties are shown in Table 1.The basic size of the suture reinforced thermal protection structure is the same as that of the test piece, and its appearance is shown in Figure 6.

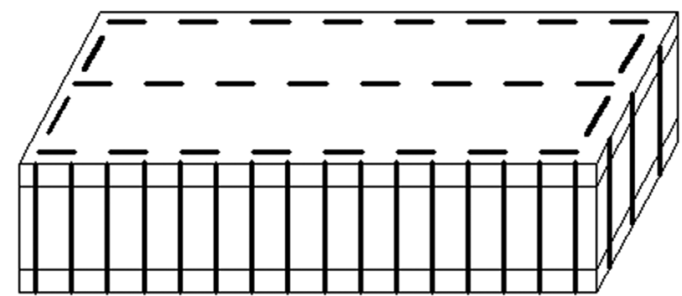

Figure 6. Sutures method for reinforced thermal protection structure.

Suture pattern 1: no stitching at all.

Suture pattern 2: firstly, the whole stitching is performed at a distance of $5 \mathrm{~mm}$ from each side of the thermal protection structure, with the needle distance of $14.5 \mathrm{~mm}$; secondly, a suture along the length Xis added at the middle of the width of the structure, and eachq stitch is $15 \mathrm{~mm}$.

Suture pattern 3: add two stitches along length $\mathrm{X}$ to stitching pattern 2.The specimen used in the test is this pattern.

Schematic diagram of different suture patterns is shown in Figure 7.
According to the loading method of the specimen and four-point bending test, the finite element model was established with the specimen on the bottom plate as the research object.The stitched sandwich structure is divided by 20 -node hexahedral elements. Titanium alloy baseplate grid division mainly uses 20-node hexahedral elements, with a small number of 10-node tetrahedral elements.

In reference ${ }^{23}$, it was pointed out that the sensitivity and convergence of the grid are related to the geometry of the grid and the size of the elements. In the finite element analysis of partition, it was necessary to ensured the good quality of the mesh. In the static structure analysis of ANSYS, good mesh quality ensuredsufficient sensitivity and good convergence. Different mesh metrics were used in ANSYS to evaluate the quality of meshes. The parallel deviation and maximum corner angle of these mesh metrics can be used to describe the geometric shape of the mesh mentioned above. In this research, "element quality" metric is applied to evaluate the mesh quality. Good sensitivity and good convergence can be acquired by good mesh quality.

After the structure was meshed, the mesh quality corresponding to different mesh is given, as shown in Figure 8. Information on the number of elements and nodes is shown in Table 2. The larger node number led to longer computation time. The calculated results of method 1 and method 2 differ by less than $5 \%$, so we believe that the mesh quality has little influence on the calculated results, which is the grid independence ${ }^{23}$. Considering the mesh quality and computing time, the global mesh size of the structure is set as $0.5 \mathrm{~mm}$ and quadratic elements are accepted.

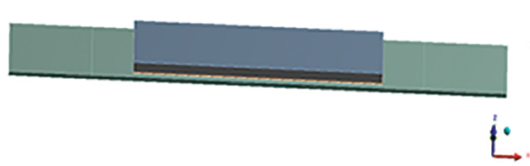

(a) suture pattern 1

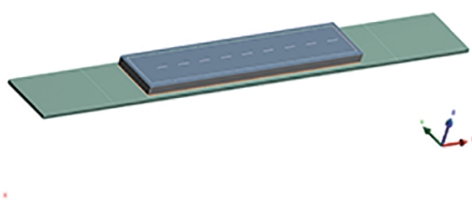

(b) suture pattern 2

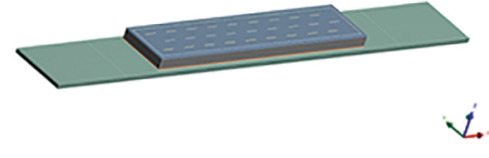

(c) suture pattern 3

Figure 7. Finite element models of suture pattern.

Table 1. Basic mechanical properties of materials.

\begin{tabular}{cccccccc}
\hline Material & $\begin{array}{c}\text { Thickness/ } \\
\mathrm{mm}\end{array}$ & $\begin{array}{c}\text { Destiny/ } \\
\left(\mathrm{g} . \mathrm{cm}^{-3}\right)\end{array}$ & $\begin{array}{c}\text { Tensile } \\
\text { passion's ratio }\end{array}$ & $\begin{array}{c}\text { Tensile } \\
\text { modulus/MPa }\end{array}$ & $\begin{array}{c}\text { Tensile } \\
\text { strength/MPa }\end{array}$ & $\begin{array}{c}\text { Bending } \\
\text { modulus/MPa }\end{array}$ & $\begin{array}{c}\text { Bending } \\
\text { strength/MPa }\end{array}$ \\
\hline Ceramic & 1 & 1.61 & 0.31 & 5230 & 53.5 & & 502 \\
Aerogel & 10 & 0.337 & 0.2 & 180 & 1 & 405.37 & 1.22 \\
Suture & & 2.6 & 0.5 & 75000 & & & \\
\hline
\end{tabular}

Table 2. Elements and nodes number with different mesh method.

\begin{tabular}{ccc}
\hline Method & Element number & Node number \\
\hline $1 \mathrm{~mm}$, Quadratic & 221625 & 992587 \\
$0.5 \mathrm{~mm}$, Quadratic & 544970 & 2619535 \\
$0.25 \mathrm{~mm}$, Quadratic & 1841635 & 9116347 \\
\hline
\end{tabular}




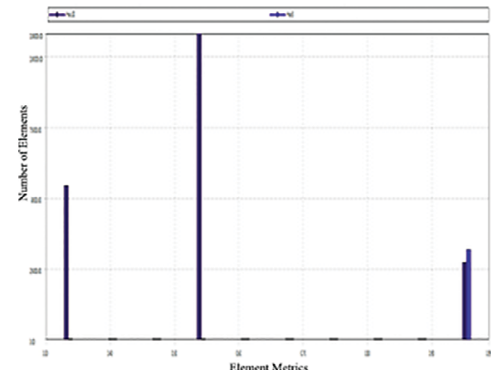

(a) $1 \mathrm{~mm}$, Quadratic

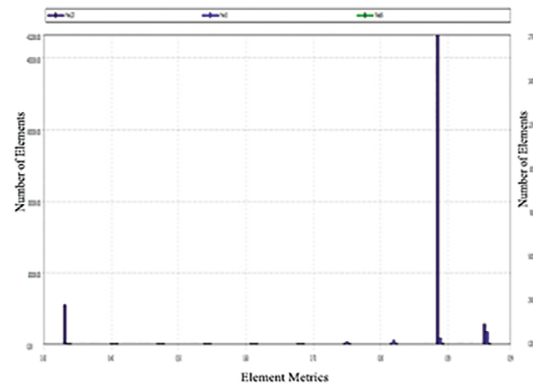

(b) $0.5 \mathrm{~mm}$, Quadratic

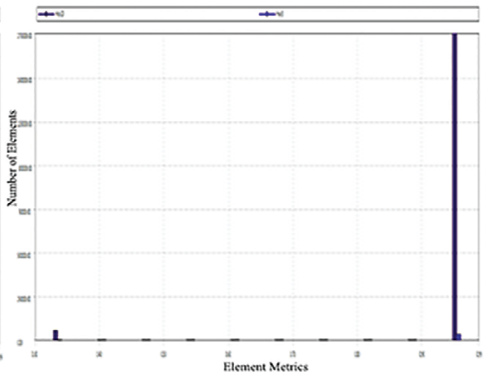

(c) $0.25 \mathrm{~mm}$, Quadratic

Figure 8. Different mesh method with mesh quality.

The strain results of the straight line (X-direction) where the strain gauge is located on the finite element model of stitched sandwich are compared with the test results, as shown inFigure 9. The deviation between the simulated value and the test value may be due to the defects of the material itself and the discreteness of the test results. The test results fit well with the finite element calculation results, and the validity of the finite element model is verified.

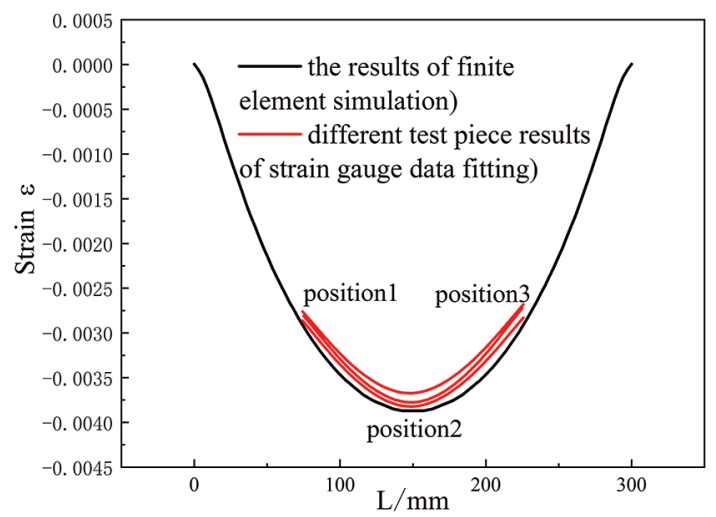

Figure 9. Comparison of experimental and simulation results.

\section{Results and Discussion}

In order to analyse the effect of sutureon the analysis models of stitched sandwich, four paths are set on the model to calculate the stress fields for analysis and discussion. The four paths are: upper panel edge path 1, lower panel edge path 2 , corner edge path 3 , and path 4 close to suture. The starting point and ending point of all paths are 1 and 2 respectively, as shown in Figure 10. Where path 4 is the corner edge of the sub-structure taken $90 \mathrm{~mm}$ from the left and $25 \mathrm{~mm}$ from the front and is located inside the overall suture.

\subsection{Tensile Properties}

\subsubsection{Finite element boundary setting}

Stage one: the suture is not considered, and the overall analysis of the stent and the test piece combination structure is performed. A tensile force of $2 * 10^{5} \mathrm{~N}$ is applied to the right side of the bracket, and the loading axial direction is at the same level as the lower surface of the structural model, and the bottom plate is deformed together with the bracket.
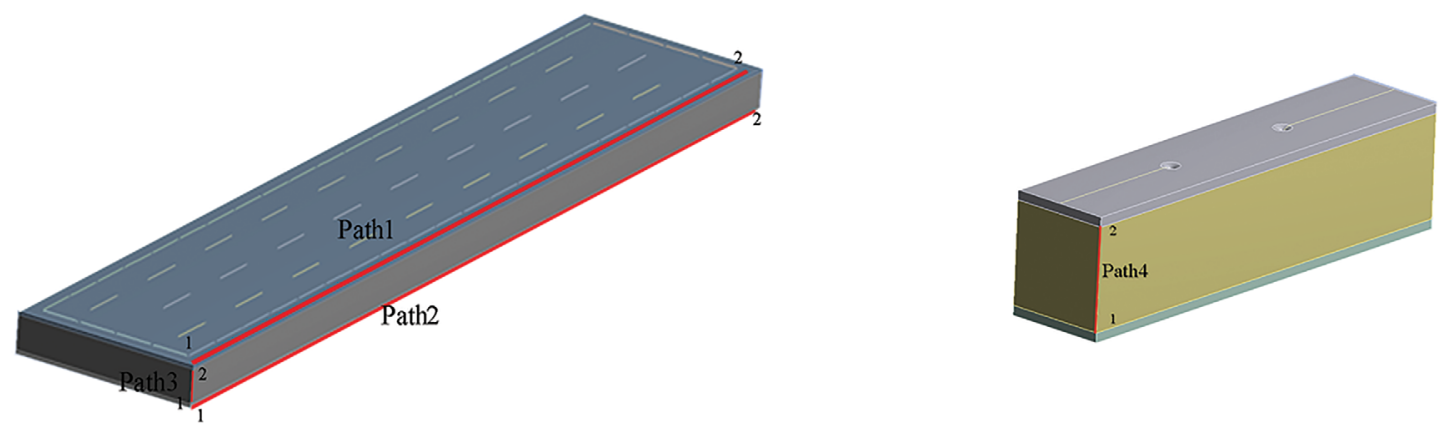

Figure 10. Four path distribution locations. 
Stage two: the bracket is removed, and the displacement of the bottom plate of the test piece calculated in the first stage is mapped to the bottom plate of the second stage as forced displacement of the second stage calculation.In the analysis,the application of $50 \mathrm{~N}$ pre-stress in suture is considered.

Stage three: based on stage two, the substructure analysis method is adopted. The analysis unit is cut out from the test piece, which the detailed structure such as punching is considered at this time.

\subsubsection{Comparison of sandwich results of different stitching patterns}

The results of suture pattern 1 and 3 models on the upper panel stress along path 1 are shown in Figure 11. When the lower panel of suture pattern 3 is pulled, its stress distribution shape is different from that of the pattern 1 opening downward, generating fluctuations in the period of the distance.The stress of the stitched sandwich increases sharply near the hole, and its wave peak is lower than the stress of the unstitched structure at the same distance. Where the hole is away from the hole, the stress of the stitched sandwich structure is much smaller than the stress of the unstitched structure at the same distance, which greatly enhances the in-plane mechanical transmission performance of the plate.The upper panel of the unstitched sandwich model and the stitched sandwich model have the maximum force in the middle part of the path 1 respectively, 3.273 $M P a$ and $3.122 \mathrm{MPa}$, respectively and the suture makes the maximum stress of the upper panel in the $\mathrm{X}$-direction decrease by about $4.6 \%$.

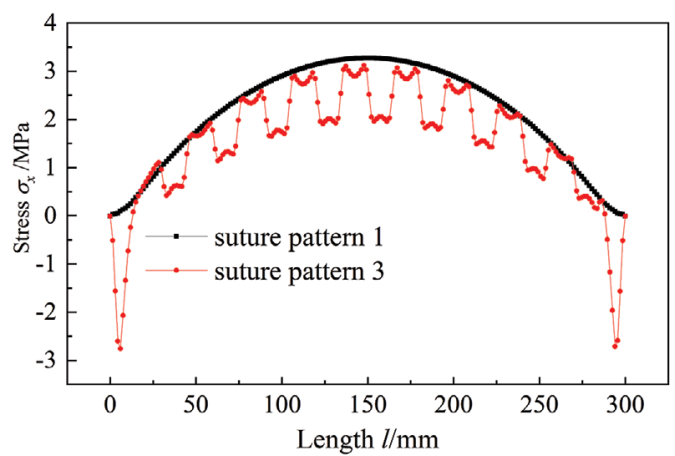

Figure 11. Curve of upper panel stress along path 1 for suture pattern 1 and 3 models.

The results of the lower panel stress on path 2 of suture pattern 1 and 3 modelsare shown in Figure 12. The stress in the pattern 3 model does not fluctuate, and the stress curve of the unstitched structure is over-fitting. It may be that the input of the second stage is the forced displacement of the first stage. The substructure with the punched hole needs to be intercepted, and the substructure is analysed in detail to ensure the accuracy of the results.

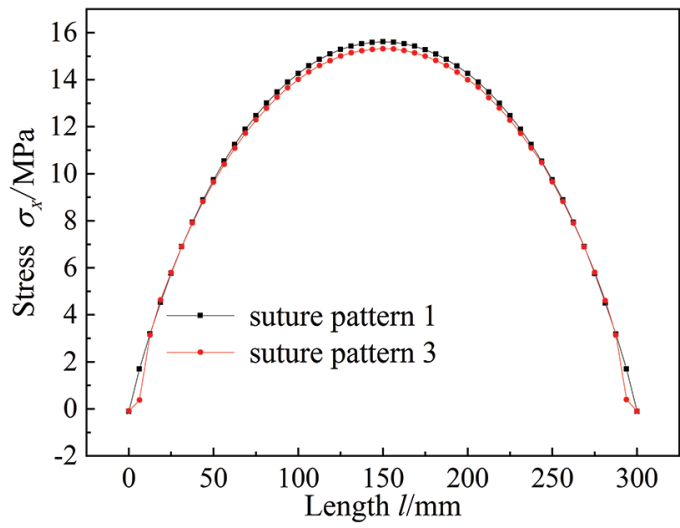

Figure 12. Curve of lower panel stress along path 2 for suture pattern 1 and 3 models.

The stress curves of the suture pattern 1and 3 models in the Z-direction along path 3 are shown in Figure 13.The bottom plate is firstly pressed and then pulledand the middle core layer is subjected to tension first. The stress tends to be steeper and then becomes gentle in the thickness range of the core layer.When the bottom layer of the stitched sandwich is uniaxially stretched, the stress of the structural tension part is smaller than that of the unstitched structure; the stress of suture pattern 3 model pressed part is greater than the suture pattern 1 model.To obtain a detailed analysis result, the core layer in the middle of the stitched sandwichstructure is remapped along the Z- direction (position same per Figure 13).

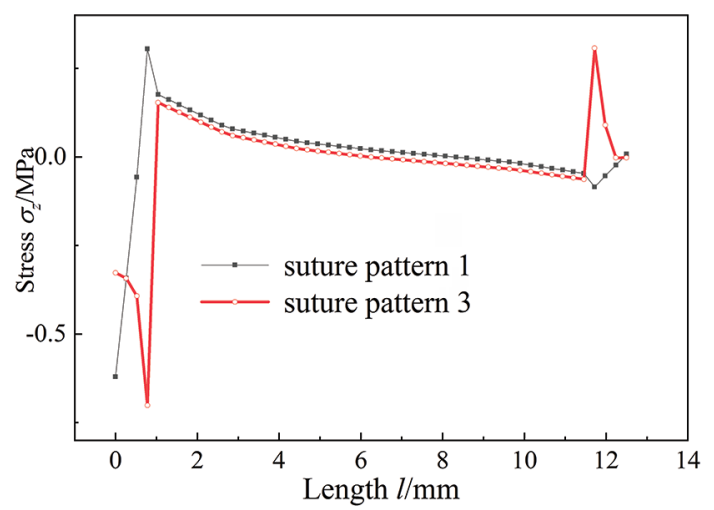

Figure 13. Curve of stress for the suture pattern 1 and 3 models along path 3 .

The stress curves of the suture pattern 3 model (core portion) along path 3 and 4 are as shown in Figure 14. It can be seen from Figure 13 that the stress of the path 4 can be approximated as a straight line without a turning point, and the stress distribution is continuously uniform; the stress of the path 3 is approximately the combination of the three-section polylineand the slope is slowed down. The size of the stitching effect on the sandwich in the Z-direction is related to the position of the suture, the closer to the suture, the greater the magnitude of the stress reduction. 


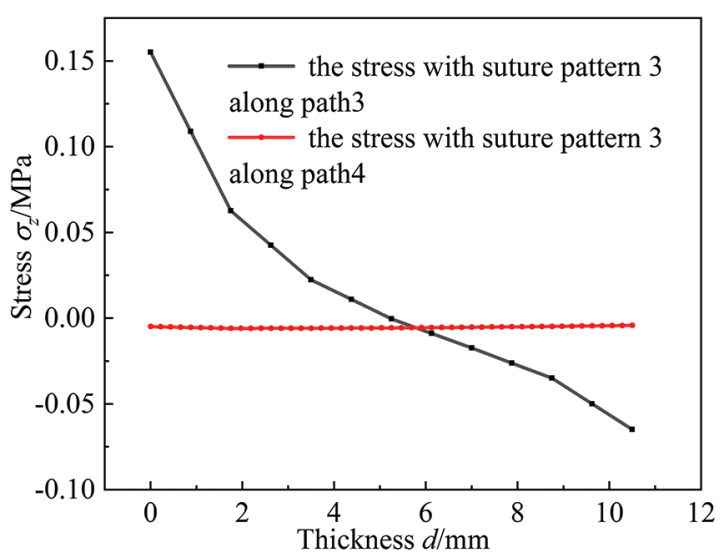

Figure 14. The curve of stress for the suture pattern 3 modelalong path3 and 4.

When the bottom plate of the suture pattern 3 modelis subjected to uniaxial stretching, the portion near the bottom plate is more affected by the suture and the stress along the Z-direction is reduced by about $30 \%$ as a whole.For the tensile part of the sandwich, the Z-direction suture is the main bearing member because the stitch modulus is much higher than the core layer.The stress of sandwich will be greatly reduced and the closer to the suture, the more obvious the effect.

The stress pattern and distribution trend of the upper and lower panels of the suture substructure along the X-direction are shown in Figure 15. In the above section, it is mentioned that the stress does not fluctuate in the suture pattern 3 model, and over-fitting with the suture pattern 1 model stress curve may be that the structural bottom plate and the clamp bracket are too tightly bonded and do not show a true fluctuation trend.In Figure 15, it can be seen that the stress distribution of the upper plate of the suture substructure and the stress distribution of the upper plate along the path 1 obtained in the second stage completely coincide, and the stress of the lower plate does not conform to the overall stress distribution obtained in the second stage, so detailed substructure analysis is an essential part of the overall structural analysis.

\subsubsection{Effect of suture density on static characteristics}

The stress distribution of the three suture patterns in the upper panel path 1 was comparedto analyse the influence of stitching density on the distribution of static characteristics as shown in Figure 16. The effect of suture on the stress reduction of thermal protection structure in the X-direction path 1 is significant, but with the increase of suture density, the overall effect of stress reduction becomes less obvious (the area comparison under each curve can be concluded).

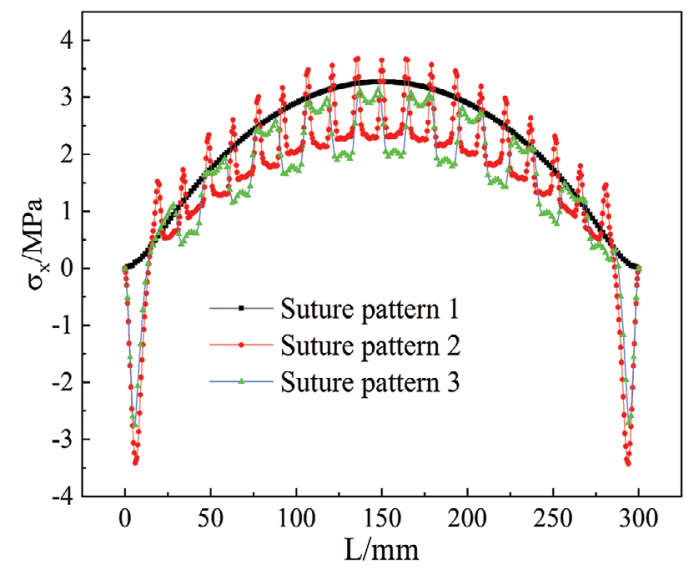

Figure 16. The distribution curve of stress on path1 under three suture patterns.

Figure 17 shows the distribution curve of stress in three suture patterns in the Z-direction path, and analyses the influence of suture density on the distribution of static characteristics.Compared with the suture pattern 1, the Z-direction overall stress of the suture pattern2 decreased by about $25 \%$, and the Z-direction stress of the suture pattern 3 decreased by about $30 \%$.It can be seen that with the increase of suture density, the overall effect of stress reduction is gradually weakened.

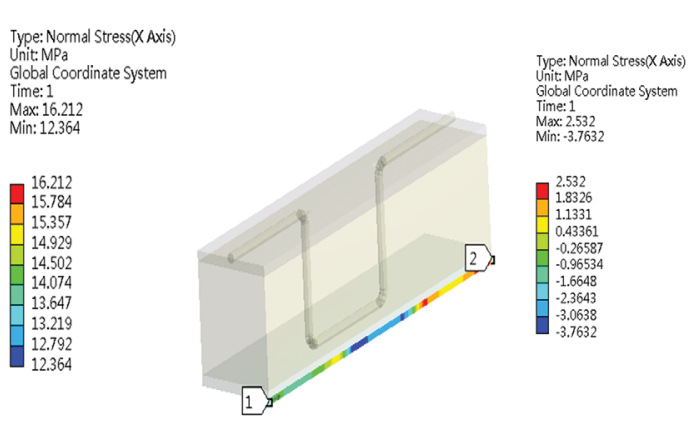

(a) path 1

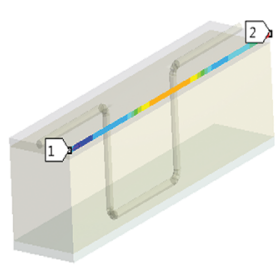

(b) path 2

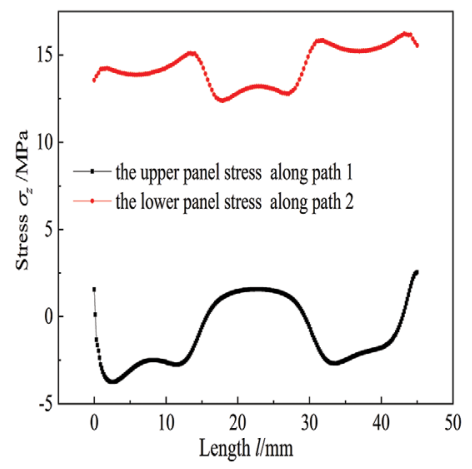

(c) path 1 and 2

Figure 15. Stress along path 1 and 2 with suturepattern 3 model substructure. 


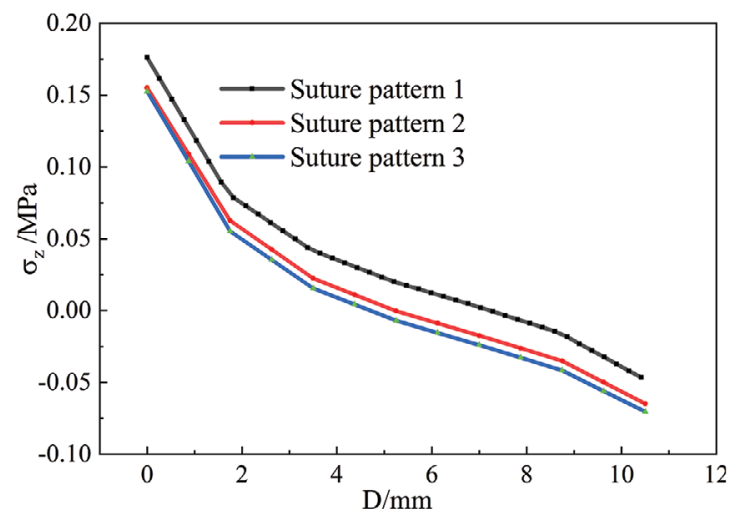

Figure 17. The distribution curve of stress on path3 under three suture patterns.

\subsection{Bending Performances}

\subsubsection{Finite element boundary setting}

Stage one:the supporting position of the bent bottom panel is located at a distance of $50 \mathrm{~mm}$ from the short side, and the left end is fixedly supported and the right end is restrained by displacement. $2000 \mathrm{~N}$ shall be applied at both ends from the short side $100 \mathrm{~mm}$, from top to bottom, and $50 \mathrm{~N}$ pre-stress shall be considered.

Stage two: based on stage one, the substructure analysis method is adopted to cut out the analysis unit from the test piece. At this time, the detailed structures such as drilling are considered.

\subsubsection{Comparison of sandwich results of different stitching patterns}

The stress of the upper panel of the sandwich with three different suture patterns along path 1,isshown in Figure18. When the stitched sandwich is subjected to four-point bending, a wide range of fluctuation with a period of distance occurs in stitched structure and the upper panel is pressed as a whole. The stress of the stitched sandwich will suddenly increase to form a trough near the holeand the value of the trough is smaller than the stress of the unstitched structure at the same distance. The stress of the upper panel of suture pattern 2 along path 1 is reduced by $11.8 \%$ compared with the unstitched structureand the stress of suture pattern 3 is reduced by $15.2 \%$. The panel is glued with the suture. When the suture is subjected to $50 \mathrm{~N}$ pretension, one side of the panel contacting with the suture is subjected to preloading.However, sutures have needle pitch: the wired part has pre-pressure and the wireless part has no pre-pressure, thus causing fluctuations.

The stress results of the bottom panel of the sandwich with three different suture patterns along path 2 are shown in Figure 19. The stress trend of the bottom panel is quite different from that of the upper paneland its distribution trend changes from steep to gentle and then to steep.

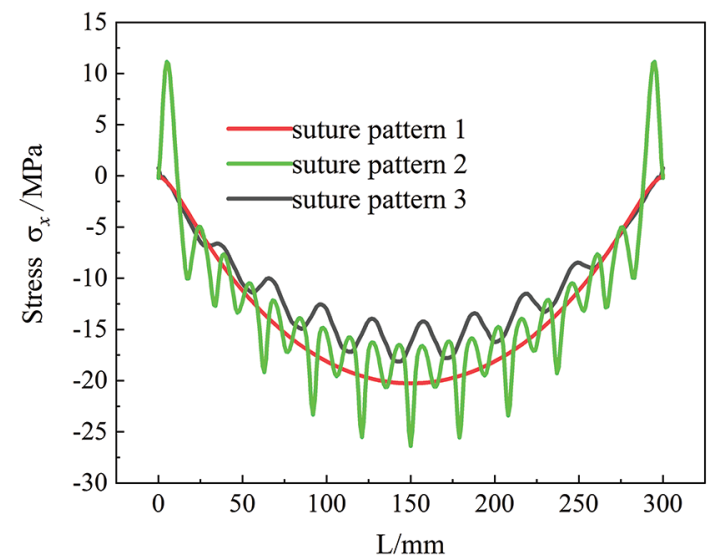

Figure 18. Three different suture patterns of sandwich on the panel stress along path 1 .

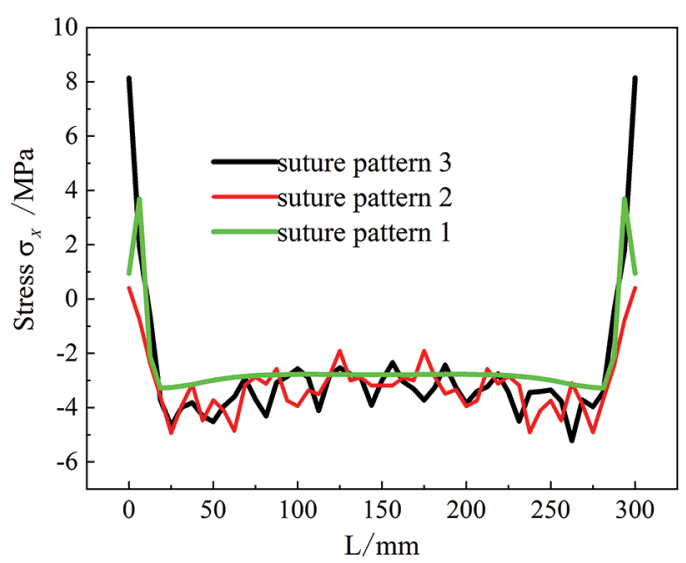

Figure 19. Three different suture patterns of sandwich on the panel stress along path2.

The bottom panel changes from tension to compression along the X-direction and then returns to tension, while the upper panel is always under compression along the $\mathrm{X}$-direction. The stress fluctuation trend of the upper panel of the unstitched pattern 1 and the stitched patterns 2 and 3 along path 2 is consistent: the stress increase from the edge to the middle and then decrease from the middle to the edge along the $\mathrm{X}$-direction.The stress of stitched sandwich will suddenly increase near the hole and the increase range is unstable. When the structure is subjected to four-point bending, the pattern with suture cannot transmit mechanical properties as compared with the pattern 1 without suture, and the deformation of the bottom panel is slightly increased due to suture.

The stress trend of the sandwich along the whole thickness is relatively complex:the change trend of the core layer in the middle of the structure along the three Z-directions of the corner path is detailed as shown in Figure 20. When the structure is subjected to four-point bending, the stress state changes from compression to tension as the height increases. 


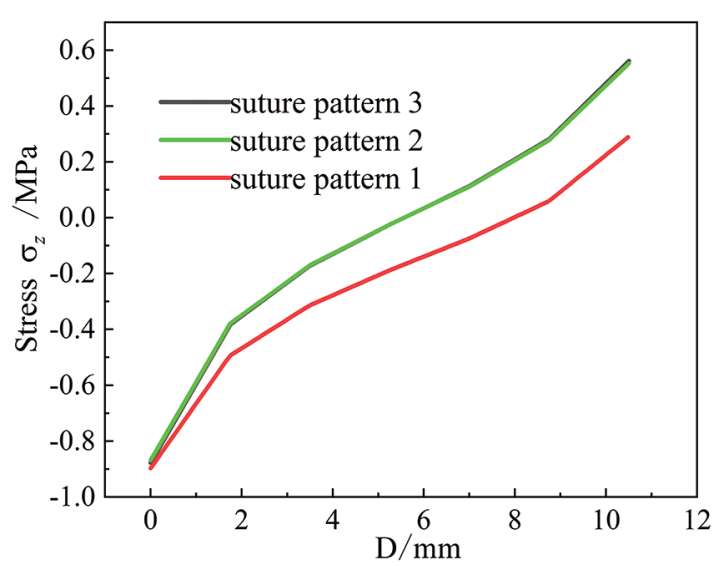

Figure 20. Three different suture patterns of sandwich structures on the panel stress and strain along path3.

In the thickness part of the core layer, the stress on path 3 are approximately the combination of three broken lines and the inclination first decreases and then increases, whilst the suture does not change the trend. The stress of the tensile part of the structure on this path is slightly lower than the stress of the unstitched structure. The distribution of suture pattern 3 and suture pattern 2 tend to coincide. It can be seen that the effect of suture density on the Z-direction mechanical properties of the corner edge outside the overall suture is limited when subjected to four-point bending.

The results of stress curves along path 4 for the sandwich of pattern 1 and pattern 3 are shown in Figure 21. The stress of the tension part close to the suture path 4 in the suture range is reduced by about $31.8 \%$ as a whole as compared with the stress without suture, thus greatly improving the Z-direction mechanical property of the internal core layer with integral suture and reducing the possibility of core layer degumming. When the core layer is pulled, because the suture modulus is much higher than the core layer aerogel, the suture along the $\mathrm{Z}$-direction is the main stress member. The stress on the core layer will naturally be greatly reduced.

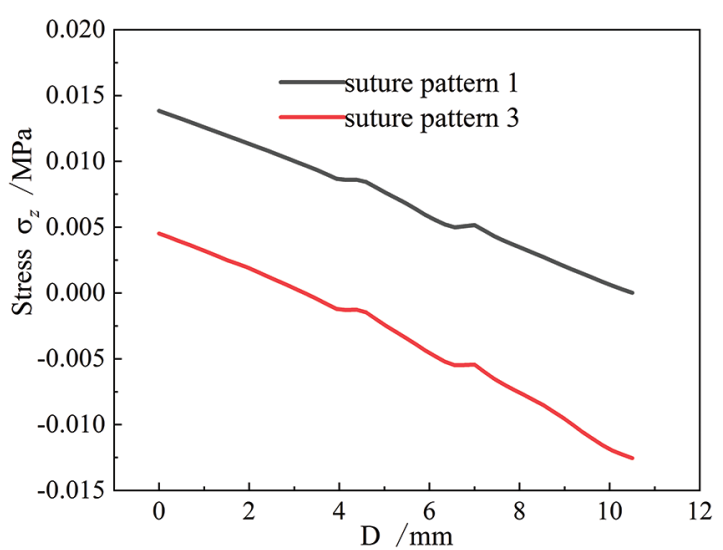

Figure 21. Two different suture patterns of sandwich structures on the panel stressalong path4.

\subsubsection{Substructure with suture structure}

The stress nephogram and distribution trend of the upper and lower plates of the suture substructure along the $\mathrm{X}$-direction are as Figure22. Drawing the stress distribution of the upper and lower panels together shows that the fourpoint bending has greater influence on the stress distribution of the upper panel, and the fluctuation trend of the lower panel can be ignored.

\section{Conclusion}

(1) With the increase of suture density, the maximum stress of the upper panel can be reduced by up to $11.5 \%$ compared with the unstitched structure, and the static performance of the thermal protection plate improves to a certain extent.

(2) Whether subjected to four-point bending or stretching, the Z-direction stress of the stitched structure decreases by about $30 \%$ as a wholeand the suture line greatly improves the vertical mechanical properties of the inner core layer of the overall suture.

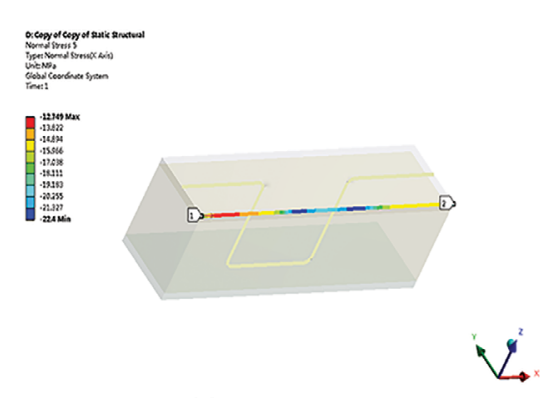

(a) path 1
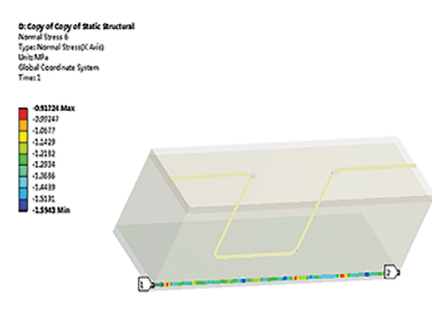

(b) path 2

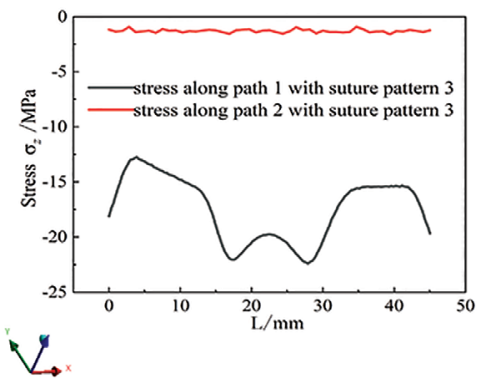

(c) path 1 and 2

Figure 22. Stress of suture substructure. 
(3) The suture does not affect the stress variation trend along the X-direction of the upper and lower panels.But it produces periodic fluctuations in distanceand the location of the fluctuation point is related to the drilling position.

(4) The enhancement effect of the suture line on the thermal protection structure in the $\mathrm{Z}$-direction is related to the location of the suture: the closer the suture is, the greater the stress reduction is.

(5) In the tensile part of the stitched thermal protection structure, the suture is the main bearing stress component, which reduces the risk of core layer degumming.

\section{References}

1. Davies JM, ed. Lightweight Sandwich Construction. London: Blackwell Science; 2001.

2. Nanayakkara AM, Feih S, Mouritz AP. Improving the fracture resistance of sandwich composite T-joints by z-pinning. Composite Structures. 2013;96:207-215.

3. Guo S, Morishima R. Numerical analysis and experiment of composite sandwich T-joints subjected to pulling load. Composite Structures. 2011;94(1):229-238.

4. Yudhanto A, Watanabe N, Iwahori Y, Hoshi H. Effect of stitch density on tensile properties and damage mechanisms of stitched carbon/epoxy composites. Composites Part B: Engineering. 2013;46:151-165.

5. Liu D. Delamination Resistance in Stitched and Unstitched Composite Plates Subjected to Impact Loading. Journal of Reinforced Plastics and Composites. 1990;9(1):59-69.

6. Francesconi L, Aymerich F. Impact damage resistance of thin stitched carbon/epoxy laminates. Journal of Physics: Conference Series. 2015;628(1):012099.

7. Lopresto V, Melito V, Leone C, Caprino G. Effect of stitches on the impact behaviour of graphite/epoxy composites. Composites Science and Technology. 2006;66(2):206-214.

8. Mouritz AP, Cox BN. A mechanistic approach to the properties of stitched laminates. Composites Part A: Applied Science and Manufacturing. 2000;31(1):1-27.

9. Yudhanto A, Iwahori Y, Watanabe N, Hoshi H. Open hole fatigue characteristics and damage growth of stitched plain weave carbon/epoxy laminates. International Journal of Fatigue. 2012;43:12-22.

10. Xie WH, Zhang BM, Du SY. Finite Element Analysis of Metallic Thermal Protection Systems Design. Acta Aeronautica et Astronautica Sinica. 2006;5:897-902. [in Chinese].
11. Meng SH, Yang Q, Xie WH, Xu CH, Jin H. Comparative Study of Structural Efficiencies of Typical Thermal Protection Concepts. AIAA Journal. 2017;55(7):2476-2480.

12. Han FY, Yan Y, Ma J. Experimental study and progressive failure analysis of stitched foam-core sandwich composites subjected to low-velocity impact. Polymer Composites. 2018;39(3):624-635.

13. Zheng XT, Zhang JF, Yang F, Chai YN, Li Y. Experimental and Analytical Study on the Mechanical Behavior of Stitched Sandwich Composite Panel with a Foam Core. Advanced Materials Research. 2008;33-37:477-482.

14. Shi SS, Chen BZ, Chen HR, Shun Z. Three-point bending and in-plane compression properties of carbon-fiber/aluminumhoneycomb sandwich panels with short-Kevlar-fiber toughening. Acta Materiae Compositae Sinica. 2017;34(9):1953-1959. [in Chinese]. DOI: 10.13801/j.cnki.fhclxb.20170104.001

15. Vaidya UK, Palazotto AN, Gummadi LNB. Low Velocity Impact and Compression-After-Impact Response of Z-Pin Reinforced Core Sandwich Composites. Journal of Engineering Materials and Technology. 2000;122(4):434-442.

16. Partridge IK, Cartié DD, Bonnington T. Manufacture and Performance of Z-Pinned Composites. In: Shonaike GO, Advani SG, eds. Advanced Polymeric Materials: Structure-Property Relationships. Boca Raton: CRC Press; 2003. p. 472-481.

17. Palazotto AN, Gummadi LNB, Vaidya UK, Herup EJ. Low velocity impact damage characteristics of Z-fiber reinforced sandwich panels -- an experimental study. Composite Structures. 1998;43(4):275-288.

18. Virakthi A, Kwon S, Lee SW, Robeson ME. Delamination resistance of composite laminated structures reinforced with angled, threaded, and anchored Z-pins. Journal of Composite Materials. 2019;53(11):1507-1519.

19. Selver E, Kaya G. Flexural properties of sandwich composite laminates reinforced with glass and carbon Z-pins. Journal of Composite Materials. 2019;53(10):1347-1359.

20. Raeisi S, Kadkhodapour J, Tovar A. Mechanical properties and energy absorbing capabilities of Z-pinned aluminum foam sandwich. Composite Structures. 2019;214:34-46.

21. Wang XY, Yang FB, Zeng JC, eds. Design principle and application of sandwich structure composites. Beijing: Chemical Industry Press Publishing; 2007.

22. Zeng JC, Wei KY, Du G, Yang JS, Ju S. Mechanical performance of integrated stitched sandwich composite. Journal of National University of Defense Technology. 2016;38:9-14. [in Chinese]. DOI: $10.11887 /$ j.cn. 201601002

23. Strang G, Fix G, eds. An Analysis of the Finite Element Method. Englewood Cliffs: Prentice-Hall Publishing; 1973. 